\title{
On the Existence of an Effective and Complete Inference System for Cryptographic Protocols
}

\author{
(Extended Abstract)
}

\author{
Liana Bozga, Cristian Ene, and Yassine Lakhnech \\ VERIMAG , 2 av. de Vignate, 38610 Grenoble, France \\ \{Liana.Bozga, Cristian.Ene, Yassine.Lakhnech\}@imag.fr
}

\begin{abstract}
A central question in the domain of program semantics and program verification is the existence of a complete inference system for assertions of the form $\pi \models \varphi$ meaning that program $\pi$ satisfies property $\varphi$. A stronger version of this question asks for an effective (decidable) complete inference system. We investigate these questions for cryptographic protocols focusing on authentication and confidentiality properties. While it is not difficult to see that a complete and effective inference system cannot exist when an unbounded number of sessions are considered, we prove that such a system exists for bounded protocols. More, precisely 1.) we provide a complete weakest pre-condition calculus for bounded cryptographic protocols and 2.) we show that assertions needed for completeness of the calculus are expressible in a decidable second order logic on terms.
\end{abstract}

\section{Introduction}

A central question in the domain of program semantics and program verification is the existence of a complete (and sound) inference system for assertions of the form $\pi \models \varphi$ meaning that program $\pi$ satisfies property $\varphi$. A stronger version of this question asks for an effective (decidable) complete inference system. This is the question of the relationship between the truth of formulae of the form $\pi \models \varphi$ and their provability. For While-programs (or counter machines), for instance, it has been proved that it is possible to design an inference system such that provability implies truth (i.e., soundness) but impossible to have a sound system that is also complete and effective, i.e., it is impossible to have a decidable inference system such that truth implies provability (see [9] for a complete survey). Roughly speaking, the reason is that one can describe transitive closures using while programs while this is not possible in general in 1st-order logics except when Peano arithmetic is included. In other words, one has to sacrifice effectiveness (e.g., by including Peano arithmetic in the logic), or completeness and accept that some valid formulae $\pi \models \varphi$ cannot be proved or even expressed. This situation of While-programs led to the what is called Cook's relative completeness: is it possible to have a complete inference system for programs, if we 
assume all facts of the underlying logic as axioms, i.e., all facts about the considered data are given? The main question we address in this paper is the following: what is the situation for cryptographic protocols?

Beyond the theoretic relevance of this question, it has several practical consequences. Indeed, if one can provide a complete inference system for cryptographic protocols this can serve as a basis to develop compositional proof theories as well as refinement theories. The latter would be of great interest as the problem of composing cryptographic protocols (CP for short), i.e., which properties are preserved when CPs are composed, as well as the relationship between the abstract specification of a $\mathrm{CP}$ and its real implementation remain two insufficiently investigated subjects (cf. [15]). Moreover, a decidable complete inference system provides a symbolic decision procedure.

In this paper, we introduce a complete and effective inference system for bounded cryptographic protocols. Let us explain what we mean. A session of a cryptographic protocol can be specified as a sequence of sending and receiving messages. One can consider either fixed bounded number of sessions or an unbounded one. In the first case, we speak about bounded protocols but in both cases the size of the messages is unbounded. It is not difficult to encode a counter machine as an unbounded CP. Hence, we know that it is not possible to have an effective complete (and sound) inference system. We show that such a system exists for bounded protocols. This provides an alternative proof of the decidability of secrecy for bounded CPs and covers more properties than in existing work. We introduce a logic, called SPL for Security Properties Logic, for describing security properties and develop a calculus for computing the weakest condition that has to be satisfied by the initial configurations of the protocol in order to guarantee that a property described by an SPL formula is satisfied. We prove soundness and completeness for the introduced calculus. Then, we study the decidability of SPL and show that although the satisfiability (existence of a model) of SPL formulae is, in general, undecidable, it is decidable for its existential fragment, i.e., the satisfiability of formulae of the form $\exists X . \varphi$, where $\varphi$ is quantifier-free can be decided effectively (Section 6). Now, it turns out that interesting security properties are expressible in the universal fragment of the logic (see Section 3.3) and that the weakest precondition of a universal formula is expressible as a universal formula (Section 5). Hence, given a protocol $\pi$ and a property $\varphi$, using the calculus one can compute a formula $w p(\pi, \varphi)$ such that there is an attack starting for an initial state satisfying $\psi$ iff $\neg w p(\pi, \varphi) \wedge \psi$ is satisfiable. Thus, if $\psi$ is given in the existential fragment, which is the interesting situation, one can effectively check whether $\neg w p(\pi, \varphi) \wedge \psi$ is satisfiable.

Related work. The results of this paper provide an algorithm for checking security properties (confidentiality and authentication) of cryptographic protocols. It has several interesting aspects:

1. it covers other properties than confidentiality (secrecy); indeed while other methods rely on an ad hoc reduction of authentication properties to secrecy, our method is directly applicable. 
2. as initial configurations are described by formulae of the introduced logic, it can deal with infinite non-regular sets of messages initially known by the intruder.

3. we believe that our method is more easily amenable to extended intruder models: in a full version, we also consider cipher block chaining.

While several methods have been designed for the verification of a fixed number of sessions [18 1331612 |7 8 to our knowledge it has not been previously proven that a decidable and complete inference system for cryptographic protocols exists.

\section{Preliminaries}

Let $X$ be a countable set of variables and let $F^{i}$ be a countable set of function symbols of arity $i$, for every $i \in \mathbb{N}$. Let $F=\bigcup_{i \in \mathbb{N}} F^{i}$. The set of terms over $X$ and $F$ is denoted by $\mathcal{T}(X, F)$. We denote by $\leq$ the subterm relation on $\mathcal{T}(X, F)$. As usual, function symbols of arity 0 are called constant symbols. Ground terms are terms with no variables. We denote by $\mathcal{T}(F)$ the set of ground terms over $F$. For any $t_{1}, t_{2} \in \mathcal{T}(X, F)$, we denote with $\mu\left(t_{1}, t_{2}\right)$ the most general unifier (shortly mgu) of $t_{1}$ and $t_{2}$, if it exists. More precisely, by $\mu\left(t_{1}, t_{2}\right)$ we denote the representation of the mgu of $t_{1}$ and $t_{2}$ as a conjunction of equalities of the form $x=t$, if it exists. If it does not exist then $\mu\left(t_{1}, t_{2}\right)$ should be the constant false (falsum). We write $t_{1} \sim t_{2}$, if $t_{1}$ and $t_{2}$ can be unified. Also, for any substitution $\sigma: X \rightarrow \mathcal{T}(X, F)$, we denote by $t \sigma$ the application to $t$ of the homomorphic extension of $\sigma$ to terms. Given a set $\tilde{x}$ of variables, we denote by $\Gamma(\tilde{x})$ the set consisting of ground substitutions with domain $\tilde{x}$. We also write $\Gamma(x)$ instead of $\Gamma(\{x\})$.

Henceforth, we tacitly identify the term $t$ with its tree representation $\operatorname{Tr}(t)$. The elements of $\operatorname{dom}(t)$ are called positions in $t$. We use $\prec$ to denote the prefix relation on $\omega^{*}$. We write $t(p)$ to denote the symbol at position $p$ in $t$ and $t_{\mid p}$ to denote the subterm of $t$ at position $p$, which corresponds to the tree $t_{\mid p}(x)=$ $t(p \cdot x)$ with $x \in \operatorname{dom}\left(t_{\mid p}\right)$ iff $p \cdot x \in \operatorname{dom}(t)$. Given a term $t$ and positions $p$ and $q$, we say that $t_{\mid p}$ dominates $t_{\mid q}$ if $p \prec q$.

If $w_{1}, w_{2} \in \Sigma^{*}$ are words over an alphabet $\Sigma$, then we denote by $w_{2}^{-1} w_{1}$ the word obtained from $w_{1}$ after removing the prefix $w_{2}$, when possible. Otherwise, $w_{2}^{-1} w_{1}$ is undefined.

\section{The Protocol and Intruder Model}

We describe in this section the model of cryptographic protocols used in this work. We mention that this model is by now a standard one used, for instance, in [4]. We begin by describing the messages involved in a protocol model. 


\subsection{Messages}

The set of messages is denoted by $\mathcal{M}$ and contains ground terms constructed from constant symbols and the function symbols encr : $\mathcal{M} \times \mathcal{K} \rightarrow \mathcal{M}$ and pair : $\mathcal{M} \times \mathcal{M} \rightarrow \mathcal{M}$, where $\mathcal{K}$ is a set of keys. Constant symbols are also called atomic messages and are defined as follows:

1. Principal names are used to refer to the principals in a protocol. The set of all principals is $\mathcal{P}$.

2. Nonces can be thought as randomly generated numbers. As their values are unpredictable, they are used to witness the freshness of a message. We denote by $\mathcal{N}$ the set of nonces.

3. Keys are used to encrypt messages. If $k$ is a key then we use $k^{-1}$ to denote its inverse. Moreover, we use $\operatorname{pbk}(A)$ to denote the public key of $A$.

For the sake of simplicity we leave out signature and hash functions but we can easily handle them in our model.

Let $\mathcal{A}=\mathcal{P} \cup \mathcal{N} \cup \mathcal{K}$ and $\mathcal{F}=\mathcal{A} \cup\{$ encr, pair $\}$. As usual, we write $\left(m_{1}, m_{2}\right)$ for $\operatorname{pair}\left(m_{1}, m_{2}\right)$ and $\{m\}_{k}$ instead of $\operatorname{encr}(m, k)$. Message terms are the elements of $\mathcal{T}(\mathcal{X}, \mathcal{F})$, that is, terms over the atoms $\mathcal{A}$, a set of variables $\mathcal{X}$ and the binary function symbols encr and pair. Messages are ground terms in $\mathcal{T}(\mathcal{X}, \mathcal{F})$, i.e, $\mathcal{M}=\mathcal{T}(\mathcal{F})$. For conciseness, we write $\mathcal{T}$ instead of $\mathcal{T}(\mathcal{X}, \mathcal{F})$.

We assume the Dolev-Yao model [10]. For obvious reasons, we refrain from recalling the model here. We use the by now standard notation $E \vdash m$ to denote the fact that the intruder can derive the message $m$ from the set $E$ of messages. A derivation of a message that does not decompose any message is denoted by $E \vdash{ }_{c} m$. We write $E \vdash M$, if $E \vdash m$ holds for every $m \in M$.

For a term $t$, we use the notation $E \forall t$ to denote that no instance of $t$ is derivable from $E$, that is, for no substitution $\sigma: \mathcal{X} \rightarrow \mathcal{M}$, we have $E \vdash t \sigma$.

We now define critical and non-critical positions in a message. The idea is that since there is no way to deduce from an encrypted message the key with which it has been encrypted, the key position in messages of the form encr $(m, k)$ is not critical 1 . Formally, given a term $t$, a position $p$ in $t$ is called non-critical, if there is a position $q$ such that $p=q \cdot 2$ and $t(q)=$ encr; otherwise it is called critical. We will also use the notation $s \in_{c} m$ to denote that $s$ appears in $m$ at a critical position, i.e., there exists $p \in \operatorname{dom}(m)$ such that $p$ is critical and $m_{\mid p}=s$.

\subsection{Process Model}

Actions are defined by:

$$
\alpha::=l \stackrel{! t}{\rightarrow} l^{\prime}\left|l \stackrel{? t(\tilde{x})}{\rightarrow} l^{\prime}\right| l \stackrel{x:=t}{\rightarrow} l^{\prime} \mid l \stackrel{x=t}{\rightarrow} l^{\prime}
$$

where $t \in \mathcal{T}$ is a term, $l, l^{\prime}$ are labels and $\tilde{x} \subseteq$ var is a set of variables. An action is an output, an input, an assignment or just an equality test. In the case of an

${ }^{1}$ For the insider, the critical position corresponds, for instance, to the subterm relation in the strand space model $[11 \mid 20]$. 
input, $\tilde{x}$ denotes the variables that are instantiated by the action. The set of actions is denoted by $\mathcal{A}$ ct. A protocol is represented by a set of sequences of actions. More precisely, a protocol $\Pi$ is given by $\sum_{i=1}^{n} \alpha_{1}^{i} \cdots \alpha_{n_{i}}^{i}$, where $\alpha_{j}^{i}=\ell_{j}^{i} \stackrel{\beta_{\dot{j}}^{i}}{\rightarrow} \ell_{j+1}^{i}$ for some $\beta_{j}^{i}$ with $j \in\left\{1, \ldots, n_{i}\right\}$. Here, the labels $\ell$ represent control points and $\sum$ is the usual non-deterministic choice. This corresponds to the representation of a fixed set of sessions put in parallel by their possible interleavings. Usually, we use the more intuitive notation: $\sum_{i=1}^{n} \ell_{0}^{i} \beta_{0}^{i} \cdots \ell_{n_{i}}^{i} \beta_{n_{i}}^{i} \ell_{n_{i}+1}^{i}$.

A configuration of a protocol run is given by a triple $\left(\sigma, E, \ell_{j}^{i}\right)$ consisting of a substitution $\sigma$, a set of messages $E$ and a control point $\ell_{j}^{i}$. The operational semantics is defined as a labelled transitional system over the set of configurations Conf. The transition relation $\left(\sigma, E, \ell_{j}^{i}\right) \stackrel{\alpha}{\longrightarrow}\left(\sigma^{\prime}, E^{\prime}, \ell_{j+1}^{i}\right)$ is defined as follows:

$-\left(\sigma, E, \ell_{j}^{i}\right) \stackrel{\alpha}{\longrightarrow}\left(\sigma, E \cup\{t \sigma\}, \ell_{j+1}^{i}\right)$, if $j \leq n_{i}$ and $\alpha=\ell_{j}^{i} \stackrel{! t}{\rightarrow} \ell_{j+1}^{i}$. That is, sending the message $t \sigma$ amounts to adding $t \sigma$ to the knowledge of the intruder.

- for $\rho \in \Gamma(\tilde{x})$ with $E \sigma \vdash t(\sigma \oplus \rho)$, we have $\left(\sigma, E, \ell_{j}^{i}\right) \stackrel{\alpha}{\longrightarrow}\left(\sigma \oplus \rho, E, \ell_{j+1}^{i}\right)$ , if $j \leq n_{i}$ and $\alpha=\ell_{j}^{i} \stackrel{? t(\tilde{x})}{\rightarrow} \ell_{j+1}^{i}$. That is, ?t corresponds to receiving any message that matches with ?t $\sigma$ and is known by the intruder.

$-\left(\sigma, E, \ell_{j}^{i}\right) \stackrel{\alpha}{\longrightarrow}\left(\sigma \oplus[x \mapsto t \sigma], E, \ell_{j+1}^{i}\right)$, if $j \leq n_{i}$ and $\alpha$ is the assignment $\ell_{j}^{i} \stackrel{x:=t}{\rightarrow} \ell_{j+1}^{i}$. The effect of an assignment is as usual.

$-\left(\sigma, E, \ell_{j}^{i}\right) \stackrel{\alpha}{\longrightarrow}\left(\sigma, E, \ell_{j+1}^{i}\right)$, if $\sigma(x)=t \sigma, j \leq n_{i}$, and $\alpha$ is the test $\ell_{j}^{i} \stackrel{x=t}{\rightarrow} \ell_{j+1}^{i}$. The action $x=t$ behaves as a filter.

The initial configuration is given by a substitution $\sigma_{0}$, a set of terms $E_{0}$ such that the variables in $E_{0}$ do not appear in the protocol description and a control point $\ell_{0} \in\left\{\ell_{0}^{1}, \cdots, \ell_{n_{0}}^{n}\right\}$.

\subsection{Expressing Security Properties}

In this subsection, we introduce an intuitive logic, which allows us to express security properties about cryptographic protocols. The purpose is to recall these properties and show how they can be described. The set of formulas $\mathcal{F}_{0}$, is defined in Table 1, $x$ is a meta-variable that ranges over the set $\mathcal{V}$ of first-order variables. First-order variables range over messages; $t$ is a meta-variable over terms. The proposition Secret $(t)$ expresses secrecy in the following (usual) sense: is true in a configuration $(\sigma, E, \ell)$, if $t \sigma$ cannot be derived by the intruder from $E \sigma$. The proposition $p c=\ell$ is true, if the program counter equals $\ell$.

Table 1. The set of formulas $\mathcal{F}_{0}$

$$
\mathcal{F}_{0} \ni \varphi, \psi::=\operatorname{Secret}(t)|x=t| p c=\ell|\top| \perp|\varphi \wedge \psi| \neg \varphi \mid \forall x \varphi
$$


Definition 1 (Semantics). The interpretation of a formula is given by the set of its models, i.e., the set $\mathcal{C}$ onf of configurations that satisfy the formula. The definition is standard except for the following clauses:

$\llbracket$ Secret $(t) \rrbracket=\{(\sigma, E, \ell) \mid E \sigma \forall t \sigma\} ; \llbracket x=t \rrbracket=\{(\sigma, E, \ell) \mid \sigma(x)=\sigma(t)\} ;$ and $\llbracket p c=\ell \rrbracket=\{(\sigma, E, \ell) \mid(\sigma, E, \ell)$ is a configuration $\}$.

There are many definitions of authentication that we can find in the literature [5, 21 14 19 17]. In the full paper, we show how these properties can be expressed in our logic.

\section{The SPL Logic}

In this section, we present a more expressive logic, the SPL logic, that embeds the logic introduced in the previous section. SPL is used in Section 5 as the underlying logic for the weakest precondition calculus.

Henceforth, let $K \subseteq \mathcal{K}$ be a fixed but arbitrary set of keys, such that $\emptyset \neq$ $K \neq \mathcal{K}$.

\subsection{A Syntactic Characterization of Secrecy}

A major problem we face for developing a complete inference system for cryptographic protocols is that secrecy. i.e., $E \forall \forall$, is not expressive enough. For instance, consider the protocol $?\{x\}_{k} ; ! x$ and the property $E \forall\left(s_{1}, s_{2}\right)$. What should be the weakest precondition that ensures this property at the end of this protocol? In this section, we introduce a modality that allows to express weakest preconditions and provides a syntactic characterization of secrecy.

Intuitively, this modality is a predicate that asserts that given the intruder's knowledge $E$, a term $s$ is protected by a key in $K$ in any message the intruder can derive from $E$.

A pair $\left(\{t\}_{k}, r\right)$, where $t$ is a term, $k \in K$ and $r$ a critical position in $\{t\}_{k}$ is called a term transducer (TT for short). Intuitively, the pair $\left(\{t\}_{k}, r\right)$ can be seen as function that takes as argument a term that matches with $\{t\}_{k}$ and returns as result the term $\{t\}_{k_{\mid r}}$. As will become clear later, a run of a CP provides the intruder with new term transducer she (he) can apply to learn new terms.

We are now ready to introduce the main modality of the logic:

Definition 2. Let $m$ and $s$ be two messages and let $w \in(\mathcal{M} \times \mathcal{P} \text { os })^{*}$ be a sequence of term transducers.

We define the predicate $m\langle w\rangle s$, which we read "s is w-protected in $m$ ", recursively on the structure of $m$ and length of $w$ :

- $m$ is atomic and $m \neq s$, or

- $m=\operatorname{pair}\left(m_{1}, m_{2}\right), m \neq s$ and both $m_{1}\langle w\rangle s$ and $m_{2}\langle w\rangle s$ are true, or

- $m=\operatorname{encr}\left(m_{1}, k\right), m \neq s, k \notin K$ and $m_{1}\langle w\rangle s$ is true, or

- $m=\operatorname{encr}\left(m_{1}, k\right), m \neq s, k \in K$ and $w=\epsilon$, or 
- $m=\operatorname{encr}\left(m_{1}, k\right), w=(b, r) . w_{1}, m \neq s, k \in K$, and $m \neq b$ or $\left.m\right|_{r}\left\langle w_{1}\right\rangle s$ is true.

This definition is easily generalized to sets of messages: Let $M$ and $S$ be sets of messages, $w$ a sequence of term transducers and $K$ a set of keys. We say that the secrets $S$ are $w$ - protected in $M$ denoted by $M\langle w\rangle S$, if it holds $\bigwedge_{m \in M, s \in S} m\langle w\rangle s$.

Example 1. Let $m=\left(\left\{A,\{N\}_{k_{1}}\right\}_{k_{2}}, A\right)$ and $K=\left\{k_{1}, k_{2}\right\}$. Then, $m\langle\epsilon\rangle N$ is true since $\left\{A,\{N\}_{k_{1}}\right\}_{k_{2}}\langle\epsilon\rangle N$ and $A\langle\epsilon\rangle N$ are true.

Let now $w=\left(\left\{A,\{N\}_{k_{1}}\right\}_{k_{2}}, 12\right) .\left(\{N\}_{k_{1}}, 1\right)$. Then, $m\langle w\rangle N$ is false since applying the term transducer $\left(\left\{A,\{N\}_{k_{1}}\right\}_{k_{2}}, 12\right)$ yields $\{N\}_{k_{1}}$ on which an application of $\left(\{N\}_{k_{1}}, 1\right)$ yields $N$.

Closure of sets of secrets. In this section, we define when a set of messages is closed. Closed sets of secrets enjoy the property that they are not derivable by composition. Intuitively, a set of messages is closed if it contains all messages along every path of the tree representing a message in the set.

Let $M$ be a set of sets of messages and let $m$ be a message. We use the notation: $m \odot M=\left\{M_{i} \cup\{m\} \mid M_{i} \in M\right\}$.

We define when a set of messages is closed. The closure of a set $S$ ensures that the intruder cannot derive a message in $S$ by composition rules:

$$
\mathrm{wc}(m)=m \odot \begin{cases}\mathrm{wc}(m 1) \cup \mathrm{wc}(m 2) & \text { if } m=(m 1, m 2) \\ \mathrm{wc}\left(m^{\prime}\right) \cup \mathrm{wc}(k) & \text { if } m=\left\{m^{\prime}\right\}_{k} \\ \left\{K^{-1}\right\} & \text { if } m \text { is atomic }\end{cases}
$$

where $K^{-1}=\left\{k^{-1} \mid k \in K\right\}$. A set $M$ of messages is called closed, if for any $m \in M$ there exists $M^{\prime} \in \operatorname{wc}(m)$ such that $M^{\prime} \subseteq M$.

Example 2. Consider the message $m=\left(\{A, N\}_{k}, B\right)$. Then wc $(m)$ consists of the following sets:

$$
\begin{array}{ll}
K^{-1} \cup\left\{\left(\{A, N\}_{k}, B\right),\{A, N\}_{k},(A, N), A\right\} & K^{-1} \cup\left\{\left(\{A, N\}_{k}, B\right),\{A, N\}_{k}, k\right\} \\
K^{-1} \cup\left\{\left(\{A, N\}_{k}, B\right),\{A, N\}_{k},(A, N), N\right\} & K^{-1} \cup\left\{\left(\{A, N\}_{k}, B\right), B\right\} .
\end{array}
$$

We can prove the following:

Lemma 1. Let $S$ be a closed set of messages. And let $E$ be a set of messages such that $S \cap E=\emptyset$. Then, $E \nvdash_{c} S$. In other words, if $S$ is closed then no message in $S$ can be derived uniquely by the composition rules.

We use the notation $E\left\langle w_{i}, S_{i}\right\rangle_{I}$ for $\bigwedge_{i \in I} E\left\langle w_{i}\right\rangle S_{i}$. Our purpose now is to define conditions on $w_{i}$ and $S_{i}$ such that for any set $E$ of messages, if $E\left\langle w_{i}, S_{i}\right\rangle_{I}$ then $m\left\langle w_{i}, S_{i}\right\rangle_{I}$, for any message $m$ derivable from $E$. In other words, such conditions ensure that $E\left\langle w_{i}, S_{i}\right\rangle_{I}$ is stable under the derivations rules defining the intruder. Remember that closure guarantees stability only under composition rules.

Example 3. Let $E=\left\{s_{1}, s_{2}\right\}$ be a set of messages. Then we have $E\langle w\rangle\left(s_{1}, s_{2}\right)$. But we have both $E \vdash\left(s_{1}, s_{2}\right)$ and $\neg\left(s_{1}, s_{2}\right)\langle w\rangle\left(s_{1}, s_{2}\right)$. 
This example shows that we need to consider only closed sets of secrets. But this is not sufficient, as showed by the following example.

Example 4. Let $E=\left\{\{s\}_{k_{1}}, k_{2}\right\}$ be a set of messages. $E\left\langle\left(\left\{\{s\}_{k_{1}}\right\}_{k_{2}}, 11\right)\right\rangle s$ is satisfied, but we have both $E \vdash\left\{\{s\}_{k_{1}}\right\}_{k_{2}}$ and $\neg\left\{\{s\}_{k_{1}}\right\}_{k_{2}}\left\langle\left(\left\{\{s\}_{k_{1}}\right\}_{k_{2}}, 11\right)\right\rangle s$.

Hence, we need to deal also with the interior term transducers. To do so, let $(b, p)$ be a term transducer. Then, we denote by $\operatorname{lpt}(b, p)$ the next term transducer in $b$ from above that dominates $b_{\mid p}$, if it exists. For lack of space we omit to give the formal definition, and we prefer to illustrate it by an example.

Example 5. Let $b$ be the term $\left\{\left(\{N\}_{k^{\prime}}, A\right)\right\}_{k}$ with $k, k^{\prime} \in K$. Then, $\operatorname{lpt}(b, 111)=$ $\left(\{N\}_{k^{\prime}}, 1\right)$. But $\operatorname{lpt}(b, 12)$ does not exist neither $\operatorname{lpt}(b, 11)$ does.

We have now everything we need to express the conditions that guarantee stability under the intruder's derivations:

Definition 3. $\left(w_{i}, S_{i}\right)_{i \in I}$ is called well-formed, if the following conditions are satisfied for every $i \in I$ :

- $S_{i}$ is closed,

- if $w_{i}=(b, r) . w$ and if there exists a term transducer $\left(b_{1}, r_{1}\right)=\operatorname{lpt}(b, r)$, then there exists $j \in I$ such that one of the following is true:

- $b \in S_{j}$

- $w_{j}=\left(b_{1}, r_{1}\right) . w$ and $S_{i} \subseteq S_{j}$.

The main property of $E\left\langle w_{i}, S_{i}\right\rangle_{I}$ is that it is stable under the intruder's deduction rules. Indeed, we have:

Proposition 1. Let $E$ be a set of messages and let $\left(w_{i}, S_{i}\right)_{i \in I}$ be well-formed such that $E\left\langle w_{i}, S_{i}\right\rangle_{I}$. Let $m$ be a message with $E \vdash m$. Then, $m\left\langle w_{i}, S_{i}\right\rangle_{I}$.

The modality $E\langle w\rangle S$ has another interesting property with respect to intruder's derivations:

Proposition 2. Let $m$ be a message and $E$ a set of messages such that $\mathcal{K} \backslash$ $K^{-1} \subseteq E$. Then, $E \forall m$ iff there exists a set of messages $A \in w c(m)$ s.t. $E\langle\epsilon\rangle A$.

\subsection{SPL: A Logic for Security Properties}

The syntax of SPL is the same as $\mathcal{F}_{0}$ except that secret $(t)$ is replaced by the following modalities: $X\langle w\rangle S$ and $x\langle w\rangle S$. Here $X$ is a fixed second-order variable, $S$ is a finite set of terms and $w$ is a finite sequence of term transducers that can contain free variables. The formulae are interpreted over a restricted set of configurations $\mathcal{C}$ on $f_{0}=\left\{(\sigma, E, l) \mid(\sigma, E, l) \in \mathcal{C}\right.$ onf, $\left.\mathcal{K} \backslash K^{-1} \subseteq E\right\}$.

Definition 4 (semantics). The semantics of SPL is defined as in Definition 1 except that we also have the following clauses:

$\llbracket X\langle w\rangle S \rrbracket=\{(\sigma, E, \ell) \mid E \sigma\langle w \sigma\rangle S \sigma\} ; \llbracket x\langle w\rangle S \rrbracket=\{(\sigma, E, \ell) \mid\{\sigma(x)\}\langle w \sigma\rangle S \sigma\}$. 
For convenience of notations, we extend the set of formulae SPL as follows:

$$
\mathrm{SPL}_{+} \ni \varphi, \psi::=\ldots|(X, x)\langle w\rangle S| t\langle w\rangle S
$$

The semantics of the newly introduced formulae is: $\llbracket t\langle w\rangle S \rrbracket=\{(\sigma, E, \ell) \mid t \sigma\langle w \sigma\rangle$ $S \sigma\} ; \llbracket(X, x)\langle w\rangle S \rrbracket=\llbracket X\langle w\rangle S \rrbracket \cap \llbracket x\langle w\rangle S \rrbracket$.

We prove that any formulae of the form $t\langle w\rangle S$ is definable in SPL.

Proposition 3. Let $s, t$ be terms, let $w$ be a sequence of term transducers and let $\mathcal{J}(t, w, s)$ be defined as follows:

$$
\mathcal{J}(t, w, s)= \begin{cases}x\langle w\rangle s & \text { if } t=x \in \mathcal{V} \\ \neg \mu(a, s) & \text { if } t=a \in \mathcal{A} \\ \mathcal{J}\left(t_{1}, w, s\right) \wedge \mathcal{J}\left(t_{2}, w, s\right) \wedge \neg \mu(t, s) & \text { if } t=\left(t_{1}, t_{2}\right) \\ \mathcal{J}\left(t_{1}, w, s\right) \wedge \neg \mu(t, s) & \text { if } t=\left\{t_{1}\right\}_{k} \wedge k \notin K \\ \neg \mu(t, s) & \text { if } t=\left\{t_{1}\right\}_{k} \wedge k \in K \wedge w=\epsilon \\ \left(\left(\mathcal{J}\left(\left.b\right|_{r}, w_{1}, s\right) \wedge \mu(b, t)\right) \vee \neg \mu(b, t)\right) & \text { if } t=\left\{t_{1}\right\}_{k} \wedge k \in K \wedge w=(b, r) . w_{1} \\ \wedge \neg \mu(t, s) & \end{cases}
$$

Then, $t\langle w\rangle s \equiv \mathcal{J}(t, w, s)$, i.e., both formulae are equivalent.

From now on, we will tacitly identify $t\langle w\rangle S$ and $\mathcal{J}(t, w, s)$. We also use the notations $(\sigma, E, \ell) \models \varphi$ for $(\sigma, E, \ell) \in \llbracket \varphi \rrbracket, t\langle\not \omega\rangle S$ for $\neg t\langle w\rangle S$, and $X\langle\not \omega\rangle S$ for $\neg X\langle w\rangle S$. Also, given $s$ a term, we write $X\langle w\rangle s$ instead of $X\langle w\rangle\{s\}$ and $t\langle w\rangle s$ instead of $t\langle w\rangle\{s\}$. We identify formulae modulo the usual properties of boolean connectives such as associativity and commutativity of $\wedge, \vee$, distributivity etc... and use $\Rightarrow$ as the classical logical implication (it can be easily defined in SPL logic using set inclusion).

The predicate Secret $(t)$ can be expressed in SPL, and hence, the specification language of Section 3.3 can be embedded into SPL.

Given a term $t$, let $F(t)$ denote the formula $\bigvee_{S^{\prime} \in w c(t)} X\langle\epsilon\rangle S^{\prime}$. Then we have:

Proposition 4. Let $t$ be a term. Then, $\llbracket$ Secret $(t) \rrbracket=\llbracket F(t) \rrbracket$.

Well-formed formulae. In Definition [3, we introduced when $\left(w_{i}, S_{i}\right)_{i \in I}$ is wellformed. As now we are dealing with formulae, we have to define when a formula is well-formed in the same sense.

Definition 5. A formula $\Phi$ is well-formed, if for any sequence of term transducers $w$ and closed set of terms $S$, whenever $\Phi \Rightarrow X\langle w\rangle S$, there exist $\left(w_{i}, S_{i}\right)_{i \in I}$ well-formed, such that $\Phi \Rightarrow \bigwedge_{i \in I} X\left\langle w_{i}\right\rangle S_{i}$ and $(w, S) \in\left(w_{i}, S_{i}\right)_{i \in I}$.

The main property satisfied by well-formed formulae is a parallel to Proposition 1 and given by the following corollary, which is a direct consequence of Definitions 3 and 5 .

Corollary 1. Let $\Phi$ be a well-formed formula such that $\Phi \Rightarrow X\langle w\rangle S$ and let $(\sigma, E, l) \in \llbracket \Phi \rrbracket$. If $m$ is a message such that $E \sigma \vdash m$, then $m\langle w \sigma\rangle S \sigma$. 
Now, the property of Corollary 1 turns out to be crucial for developing a complete weakest precondition calculus and well-formedness has to be preserved. Therefore, we introduce the function $\mathcal{H}$. It takes as arguments a formula $X\langle b . w\rangle S$ and computes the weakest (the largest w.r.t. set inclusion) well-formed formula (see Definition 5) $\mathcal{H}(X\langle b . w\rangle S)$, such that $\mathcal{H}(X\langle b . w\rangle S) \Rightarrow X\langle b . w\rangle S$ :

$$
\mathcal{H}(X\langle b . w\rangle S)= \begin{cases}X\langle b . w\rangle S & \text { if } \operatorname{lpt}(b) \text { is undefined } \\ X\langle b . w\rangle S \wedge & \\ \left(\mathcal{H}\left(X\left\langle b_{1} . w\right\rangle S\right) \vee \bigvee_{S^{\prime} \in w c(t)} X\langle\epsilon\rangle S^{\prime}\right) & \text { if } b=(t, p) \wedge b_{1}=\operatorname{lpt}(b)\end{cases}
$$

Proposition 5. Let $\Phi$ be a well-formed formula. Let b.w be a sequence of term transducers and $S$ a closed set of terms such that $\Phi \Rightarrow X\langle b . w\rangle S$. Then $\Phi \Rightarrow$ $\mathcal{H}(X\langle b . w\rangle S)$.

\section{$5 \quad$ Weakest Precondition Calculus}

We are interested in proving partial correctness of bounded cryptographic protocols w.r.t. pre- and post-condition given by universally quantified spLformulae. Thus, using the usual notation, we are interested in proving validity of Hoare triples $\{\varphi\} \pi\{\psi\}$. As our formalization of bounded CP consists of the actions, sequential composition and non-deterministic choice, the Hoare logic contains axioms for the actions and the usual inference rules for composition and choice, and the Consequence rule. The rules are standard. Therefore, we focus on the axioms for the actions. That is, for each action we show that we can express the weakest liberal precondition in SPL.

Let us now precisely define the fragment of SPL for which we develop a complete Hoare Logic. As shown in Section 3.3 most security properties (authentication and secrecy at least) can be expressed by such formulae. We denote this fragment by $\mathrm{SPL}_{\forall}$.

$$
\varphi, \psi::=X\langle w\rangle S|(X, x)\langle w\rangle S| x=t|p c=\ell| x \neq t|\top| \perp|\varphi \wedge \psi| \varphi \vee \psi \mid \forall \tilde{x} \varphi
$$

The weakest precondition of a set of configurations $\mathcal{C} \subseteq \mathcal{C}$ onf with respect to an action $\alpha$, denoted $w \ln (\alpha, \mathcal{C})$ is defined to be the set of configurations $s$, such that whenever action $\alpha$ is allowed in $s$, it leads to a configuration in $\mathcal{C}$. More formally

$$
w l p(\alpha, \mathcal{C})::=\left\{(\sigma, E, l) \mid(\sigma, E, l) \stackrel{\alpha}{\longrightarrow}\left(\sigma^{\prime}, E^{\prime}, l^{\prime}\right) \Rightarrow\left(\sigma^{\prime}, E^{\prime}, l^{\prime}\right) \in \mathcal{C}\right\} .
$$

Given a formula $\varphi$, we use $w l p(\alpha, \varphi)$ instead of $w l p(\alpha, \llbracket \varphi \rrbracket)$ to denote the weakest precondition of a formula $\varphi \in$ SPL.

Let $t$ be a term and $p$ a valid position in $t$. Then, we denote by $\operatorname{lpp}(t, p)$ the position of the first term transducer in $t$ from above that dominates $p$ if it exists. For lack of space we omit to give the formal definition, and we prefer to illustrate it by an example. 
Example 6. Consider the term $t=\left(\left\{A,\{N\}_{k_{1}}\right\}_{k_{2}}, N\right)$, where $k_{1}, k_{2} \in K$. Let $p=1121$ and $p^{\prime}=2$. Thus, $t_{\mid p}=t_{\mid p^{\prime}}=N$. Then, we have $\operatorname{lpp}(t, p)=1$, which corresponds to the key $k_{2} ; \operatorname{lpp}\left(t, p^{\prime}\right)$ is, however, undefined.

We remind from section 4 , that given a term $t, F(t)::=\bigvee_{S^{\prime} \in w c(t)} X\langle\epsilon\rangle S^{\prime}$. The intuitive explanation of the lemma is the following: being in a state $(\sigma, E, l)$, in order to be able to make an input $t(\tilde{x})$, such that $\tilde{x}$ are instantiated by $\rho$, it must be that $(\sigma, E, l) \notin \llbracket F(t \rho) \rrbracket$.

Lemma 2. Let $E$ be a set of terms, $l$ be a label and let $\rho$ and $\sigma$ be ground substitutions such that $\operatorname{dom}(\rho)=\tilde{x}$ and $(\operatorname{dom}(\sigma) \cup \operatorname{var}(E)) \cap \tilde{x}=\emptyset$. Then it holds $(\sigma, E, l) \in \llbracket F(t \rho) \rrbracket$ iff $E \sigma \not \forall t(\sigma \oplus \rho)$.

Let $t$ be a term, $w$ a sequence of term transducers and $S$ a set of terms. We denote by $\mathcal{G}(t, w, S)$ the formula obtained from $\bigwedge_{s \in S} \mathcal{J}(t, w, s)$ as follows:

- First, use distributivity of $\wedge$ and $\vee$ to push "inside" $\bigwedge_{s \in S}$ as much as possible.

- Then, replace any occurrence of $\bigwedge_{s \in S} x\langle w\rangle s$ by $(X, x)\langle w\rangle S$.

It is easy to prove by induction on the structure of $t$ that $\mathcal{G}(t, w, S) \in \mathrm{SPL}_{\forall}$, and similar to Proposition[3, we can prove that $X\langle w\rangle S \wedge \mathcal{G}(t, w, S) \equiv X\langle w\rangle S \wedge t\langle w\rangle S$.

Lemma 3 gives the weakest condition that has to be satisfied in a configuration $s$, such that if in the next step $x$ is instantiated by an input ?t $(\tilde{x})$, the reached configuration $s^{\prime}$ satisfies $x\langle w\rangle S$. The key idea can be explained by considering the sequence of actions ?t $(\tilde{x}) ; ! x$. That is, if a secret $s$ that appears in $x$ has to be protected then it has to appear in $x$ under an encryption. Thus, before executing ?t( $\tilde{x}) ; ! x$, it should be the case that even if we provide the intruder with the term transducer that takes as input $t(\tilde{x})$ and yields $x$, it is not possible to derive $s$.

Lemma 3. Let $t$ be a term, $S$ a set of terms, $w$ a sequence of term transducers, $x$ a variable and $P_{x, t}$ the set of critical positions of $x$ in $t$. Let

$$
\mathcal{K}(t, x, w, S)=X\langle w\rangle S \wedge \bigwedge_{p=l p p\left(t, p_{x}\right), p_{x} \in P_{x, t}} \mathcal{H}\left(X\left\langle\left(\left.t\right|_{p}, p^{-1} p_{x}\right) . w\right\rangle S\right)
$$

Let $E$ be a set of terms, $l$ and $l^{\prime}$ labels, and $\rho, \sigma$ ground substitutions such that $\operatorname{dom}(\rho)=\tilde{x}, x \in \tilde{x},(\operatorname{dom}(\sigma) \cup \operatorname{var}(E)) \cap \tilde{x}=\emptyset$. Let $\Phi$ a well-formed formula such that whenever $E \sigma \vdash t(\sigma \oplus \rho)$, it holds

$$
\left(\sigma \oplus \rho, E, l^{\prime}\right) \in \llbracket(X, x)\langle w\rangle S \rrbracket \text { iff }(\sigma, E, l) \in \llbracket \Phi \rrbracket
$$

Then $\llbracket \Phi \rrbracket=\llbracket \rho(\mathcal{K}(t, x, w, S)) \rrbracket$.

Now we are ready to introduce the weakest preconditions for all formulae in $\mathrm{SPL}_{\forall}$. Remark that in the case of input, $F(t)$ is used for partial correctness: if an input ?t $(\tilde{x})$ is not allowed in a configuration $s$ (i.e. it holds $s \in \llbracket F(t) \rrbracket$ ), then for any $\varphi$, we have that $s \in w l p(? t(\tilde{x}), \varphi)$. 
Definition 6 (definition of wlp). The function wlp, which gives the weakest preconditions for $\mathrm{SPL}_{\forall}$, is defined below:
1. $\hat{w l} p\left(l \stackrel{! t}{\rightarrow} l^{\prime}, \varphi\right) \stackrel{\text { def }}{=} p c=\ell \Rightarrow \varphi \wedge \mathcal{G}(t, w, S)$
2. $\hat{w l} p\left(l \stackrel{! t}{\rightarrow} l^{\prime}, \varphi\right) \stackrel{\text { def }}{=} p c=\ell \Rightarrow \varphi$
if $\varphi \in\{X\langle w\rangle S,(X, x)\langle w\rangle S\}$
2. $w i p(l \rightarrow l(\tilde{x}), \varphi)=p c=\ell \Rightarrow \varphi$
if $\varphi \in\left\{x \neq t^{\prime}, x=t^{\prime}, \top, \perp\right\}$
3. $\hat{w l} p\left(l \stackrel{\text { ?t(x) }}{\rightarrow} l^{\prime},(X, x)\langle w\rangle S\right) \stackrel{\text { def }}{=} p c=\ell \Rightarrow(F(t) \vee \mathcal{K}(t, x, w, S)) \quad$ if $x \in \tilde{x}$
4. $\hat{w l} p\left(l \stackrel{? t(\tilde{x})}{\rightarrow} l^{\prime}, \varphi\right) \stackrel{\text { def }}{=} p c=\ell \Rightarrow(F(t) \vee \varphi)$
$\left.t^{\prime}, x=t^{\prime}, \top, \perp\right\}$ and $y \notin \tilde{x}$
5. $\hat{w l} p\left(l \stackrel{x:=t}{\rightarrow} l^{\prime}, \varphi\right) \stackrel{\text { def }}{=} p c=\ell \Rightarrow \varphi[t \sigma / x]$ $\left.t^{\prime}, x=t^{\prime}, \top, \perp\right\}$
6. $\hat{w l} p\left(l \stackrel{x=t}{\rightarrow} l^{\prime}, \varphi\right) \stackrel{\text { def }}{=} p c=\ell \Rightarrow(\sigma(x)=t \sigma \Rightarrow \varphi)$ if $\varphi \in\{X\langle w\rangle S,(X, y)\langle w\rangle S, x \neq$ $\varphi \in\left\{X\langle w\rangle S,(X, x)\langle w\rangle S, x \neq t^{\prime}, x=t^{\prime}, \top, \perp\right\}$
7. $\hat{w l} p\left(l \stackrel{\beta}{\rightarrow} l^{\prime}, p c=l^{\prime \prime}\right) \stackrel{\text { def }}{=} p c=\ell \Rightarrow \ell^{\prime}=\ell^{\prime \prime}$
8. $\hat{w l p}(\alpha, \varphi \vee \psi) \stackrel{\text { def }}{=} \hat{w l} p(\alpha, \varphi) \vee w \hat{l} p(\alpha, \psi)$
9. $\hat{w l} p(\alpha, \varphi \wedge \psi) \stackrel{\text { def }}{=} \hat{w l} p(\alpha, \varphi) \wedge \hat{w l} p(\alpha, \psi)$
10. $\hat{w l} p(\alpha, \forall \tilde{x} \varphi) \stackrel{\text { def }}{=} \forall \tilde{x} \cdot \hat{w l} p(\alpha, \varphi)$

$$
\text { if } \operatorname{var}(\alpha) \cap \tilde{x}=\emptyset
$$

It is easy to see that for any formula $\varphi \in \mathrm{SPL}_{\forall}$ and any action $\alpha$, wlp $(\alpha, \varphi) \in$ $\operatorname{SPL}_{\forall}$. Then, we define the formula $\operatorname{WLP}(\alpha, \varphi)$ as follows: $\operatorname{WLP}(\alpha, \varphi)=\hat{w l} p(\alpha, \varphi)$, if $\alpha \neq l \stackrel{? t(\tilde{x})}{\rightarrow} l^{\prime}$ and $\operatorname{wLP}\left(l \stackrel{? t(\tilde{x})}{\rightarrow} l^{\prime}, \varphi\right)=\forall \tilde{x} \cdot w \hat{l} p\left(l \stackrel{? t(\tilde{x})}{\rightarrow} l^{\prime}, \varphi\right)$.

Then, we have the following theorem:

Theorem 1. The wp-calculus of Definition 6 is sound and complete. I.e., let $\alpha$ be any action and $\varphi$ any formula in $\operatorname{SPL} \forall$. Then, $w l p(\alpha, \llbracket \varphi \rrbracket)=\llbracket \operatorname{WLP}(\alpha, \varphi) \rrbracket$.

Hence, following the usual completeness proof for Hoare logic, we can prove:

Corollary 2. The Hoare logic consisting of the inference rules for composition, choice and consequence and the axiom schema $\{\operatorname{WLP}(\alpha, \varphi)\} \alpha\{\varphi\}$, for each action, is sound and complete.

\section{Decidability of SPL}

In this section, we study the decidability of the existence of a model (the satisfiability problem) of an SPL formula. We prove decidability of this problem for existential formulae (i.e., formulae in $\Sigma_{0}$ ) and undecidability in the general case. Notice that since we showed in Section 5 that given a formula $\varphi$ in SPL $\forall$ and a bounded $\mathrm{CP} \pi$, one can compute $\operatorname{wLP}(\pi, \varphi)$, decidability of the satisfiability of existential formulae yields a decision procedure. Indeed, assume that we are given an existential formula $\psi$ and $\varphi$ in $\operatorname{SPL}_{\forall}$, assume also that we are given a bounded CP $\pi$ then $\{\psi\} \pi\{\varphi\}$ is true iff $\psi \wedge \neg \operatorname{WLP}(\pi, \varphi)$ is not satisfiable. Notice also that undecidability of SPL entails the non-existence of a complete and effective Hoare logic for bounded CP and SPL.

To prove decidability for existential formulae we follow a rule based approach (e.g., [136] for two nice surveys) i.e.: 
1. We introduce a set of formulae in solved form. For these formulae it is easy to decide whether a model exists.

2. We introduce a set of rewriting rules to transform any formula in the existential fragment into a solved form.

3. We prove soundness of these rules.

4. We also prove their completeness, i.e, termination for a given control that normal forms are indeed in solved form.

We will encounter two sorts of rewriting rules:

- Deterministic rules are of the form $\varphi \rightarrow \varphi^{\prime}$. They transform a given problem into a single problem. A deterministic rule is sound, if $\llbracket \varphi \rrbracket=\llbracket \varphi^{\prime} \rrbracket$.

- Non-deterministic rules of the form $\varphi \rightarrow \varphi_{1}, \cdots, \varphi_{n}$. They transform a given problem into a set of problems. A non-deterministic rule is sound, if $\llbracket \varphi \rrbracket=$ $\bigcup_{i=1}^{n} \llbracket \varphi_{i} \rrbracket$

In this section, we do not consider formulae of the from $p c=\ell$. It will be clear that adding these formulae does not add any technical difficulty; it is only cumbersome to consider them here.

Thus, given a formulae $\varphi$ with $x_{1}, \cdots, x_{n}$ as free variables, a model of $\varphi$ is pair $(\sigma, E)$ consisting of a ground substitution $\sigma$ over $x_{1}, \cdots, x_{n}$ and a set $E$ of messages.

\subsection{Decidability of $\Sigma_{0}$ Formulae}

Let $\psi$ be a formula in SPL of the form $\exists x_{1}, \cdots, x_{n} \cdot \varphi$, where $\varphi$ is a conjunction of literals, i.e., $X\langle w\rangle S|x\langle w\rangle S| x=t|\top| X\langle\not w\rangle S|x\langle\not w\rangle S| x \neq t \mid \perp$, with $x_{1}, \cdots, x_{n}$ as first-order free variables.

Notice that the satisfiability of any formula $\exists x_{1}, \cdots, x_{n} \cdot \varphi$, where $\varphi$ is quantifier-free can be reduced to a finite set of satisfiability problems of formulae in the form above.

Solved form. A formula is called in solved form if is syntactically equal to $\top$, $\perp$ or $\exists x_{1}, \cdots, x_{n} \cdot \varphi$ and $\varphi$ is of the form:

$$
\bigwedge_{i=1}^{n}\left[\bigwedge_{j=1}^{m_{i}} x_{i}\langle\epsilon\rangle t_{i}^{j} \wedge \bigwedge_{j=1}^{l_{i}} x_{i}\langle\notin\rangle u_{i}^{j} \wedge \bigwedge_{j=1}^{o_{i}} x_{i} \neq v_{i}^{j}\right] \text { such that: }
$$

1.) For any $i=1, \cdots, n, x_{i} \notin \operatorname{var}\left(t_{i}^{j}\right), x_{i} \notin \operatorname{var}\left(u_{i}^{j}\right)$, and $x_{i} \notin \operatorname{var}\left(v_{i}^{j}\right)$ and 2.) There is an ordering $x_{i_{1}}, \cdots, x_{i_{n}}$ of $x_{1}, \cdots, x_{n}$ such that the intersection of $\bigcup_{k=1}^{l_{i_{k}}} \operatorname{var}\left(u_{i_{k}}^{k}\right)$ with $\left\{x_{i_{k+1}}, \cdots, x_{i_{n}}\right\}$ is empty.

We now show how one can "easily" check whether a formula in solved form has a model. We only consider the third type of solved formulae. So, let $\varphi$ a conjunction as above. We define a particular substitution $\sigma$ such that $\varphi$ has a 
model iff it is satisfied by $\sigma$. To do so, let $k \in K$ be a fixed key. Let $F(n)$, for $n \geq 1$, denote $n$ concatenations of $k$, i.e., $F(1)=k$ and $F(n+1)=\operatorname{pair}(k, F(n))$. Let now $N$ be a natural number strictly bigger than the size of the formula $\varphi$. We then define the substitution $\sigma$ recursively as follows:

1.) If $n=1$, i.e., there is only one variable then $\sigma\left(x_{i_{1}}\right)=\left(u_{i_{1}}^{1},\left(\cdots,\left(u_{i_{1}}^{l_{i_{1}}}\right.\right.\right.$, $\left.\left.\left.\left\{F\left(N+i_{1}\right)\right\}_{k}\right) \cdots\right)\right)$. In case $l_{i_{1}}=0$ this term is understood as $\left\{F\left(N+i_{1}\right)\right\}_{k}$.

2.) If $n>1$ then replace $x_{i_{1}}$ by $\sigma\left(x_{i_{1}}\right)$ in $\varphi$. This yields a new formula $\varphi^{\prime}$ and the ordering $x_{i_{2}}, \cdots, x_{i_{n}}$, and by recursion, a substitution $\sigma^{\prime}$. Then, let $\left.\sigma=\left[x_{i_{1}} \mapsto\left(u_{i_{1}}^{1} \sigma^{\prime},\left(\cdots,\left(u_{i_{1}}^{l_{i_{1}}} \sigma^{\prime},\left\{F\left(N+i_{1}\right)\right)\right\}_{k}\right) \cdots\right)\right)\right] \oplus \sigma^{\prime}$.

Theorem 2. Let $\varphi$ be a formula in solved form syntactically different from $\top$ and $\perp$. Let $\sigma$ be the substitution as defined above. Then, $\varphi$ has a model iff $\sigma$ satisfies $\varphi$.

Table 2. Rules for transformations into a solved form

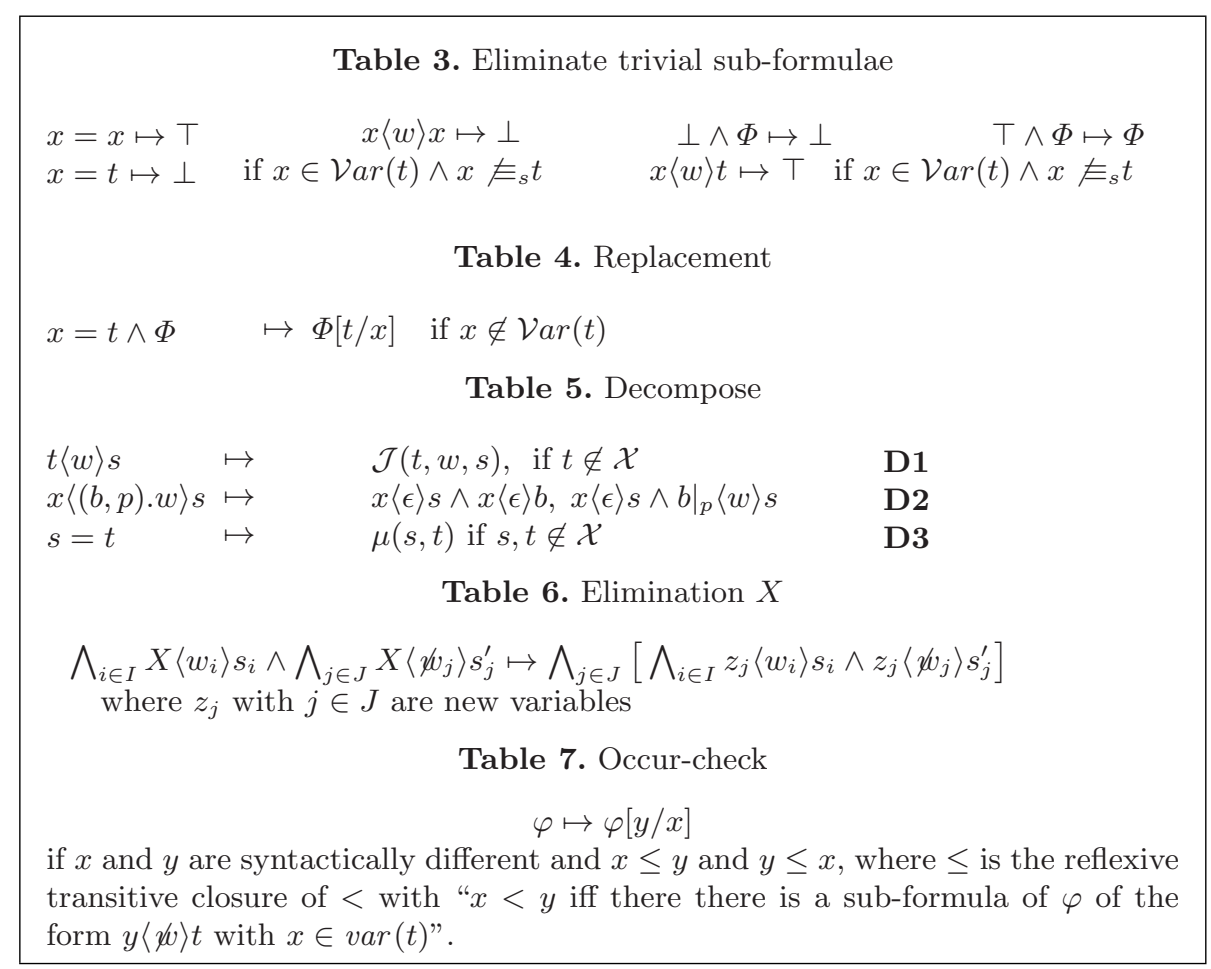

Theorem 3. Application of the rules of Table 2 terminates in a solved form.

In this table, for the rules of the form $\varphi \longrightarrow \psi$, where $\varphi$ is an atomic formula $(s=t$ or $s s\langle w\rangle s)$, we tacitly assume a rule $\neg \varphi \longrightarrow \neg \psi$. Even more, we suppose that $\neg \psi$ is represented a set of formulae in conjunctive normal form. 
If we allow both existential and universal quantifiers, then the decision problem becomes undecidable. Indeed, we can show that Post's correspondence problem is reducible to the decision problem in our logic.

Theorem 4. Post's correspondence problem is reducible to the decision problem for the SPL logic.

\section{Conclusions}

We showed that it is possible to have a complete and effective Hoare Logic for bounded cryptographic protocols and an expressive assertion language. This assertion language allows to specify secrecy as well as authentication and other properties. As a consequence of this result, we have a decision procedure for bounded cryptographic protocols and a large class of security properties allowing an infinite set of messages initially known by the intruder. The latter point might seem minor but is not. Indeed, if we are interested in composing protocols we have to take into account that we have no bound on how many sessions have taken place before, and hence, we should allow infinite sets of messages. Thus, in this paper, besides developing (to our knowledge) for the first time a result concerning the existence of an effective and complete Hoare Logic for CP, we significantly extend existing decidability results in two directions: 1.) larger class of properties and 2.) more general initial conditions. We also believe that this paper presents a general framework for a uniform presentation of different decidability results for bounded CP with weaker cryptographic hypothesis, e.g., considering equational theories. In the full paper, we develop this point of view for Cipher Block Chaining and for the xor-theory.

The method presented in this paper is a basis for analyzing unbounded protocols using approximations as those used in [4], where widening is used to guarantee termination. The interesting results of [2] can be used to restrict the use of the widening operator, and hence, obtain more precise analysis.

\section{References}

1. R. M. Amadio and D. Lugiez. On the reachability problem in cryptographic protocols. In International Conference on Concurrency Theory, volume 1877 of LNCS, pages 380-394, 2000.

2. B. Blanchet and A. Podelski. Verification of cryptographic protocols: Tagging enforces termination. In Andrew D. Gordon, editor, FoSSaCS'03: Foundations of Software Science and Computation Structures, volume 2620 of LNCS, pages 136152. Springer, 2003.

3. M. Boreale. Symbolic trace analysis of cryptographic protocols. In ICALP: Annual International Colloquium on Automata, Languages and Programming, 2001.

4. L. Bozga, Y. Lakhnech, and M. Périn. Abstract interpretation for secrecy using patterns. In TACAS'03, volume 2619 of LNCS, 2003.

5. M. Burrows, M. Abadi, and R. Needham. A logic of authentication. ACM Transactions on Computer Systems, 8(1):18-36, February 1990. 
6. H. Comon. Disunification: A survey. In Computational Logic: Essays in Honor of Alan Robinson. MIT Press, Cambridge, MA, 1991.

7. H. Comon and V. Shmatikov. Is it possible to decide whether a cryptographic protocol is secure or not? Journal of Telecommunications and Information Technology, 2002.

8. H. Comon-Lundh and V. Cortier. New decidability results for fragments of firstorder logic and application to cryptographic protocols. In 14th Int. Conf. Rewriting Techniques and Applications (RTA'2003), volume 2706 of LNCS, 2003.

9. P. Cousot. Methods and Logics for Proving Programs. In Handbook of Theoretical Computer Science, Volume B: Formal Methods and Semantics, pages 841-994. Elsevier Science Publishers B. V., 1990.

10. D. Dolev and A. C. Yao. On the security of public key protocols. IEEE Transactions on Information Theory, 29(2):198-208, 1983.

11. F.J.T. Fábrega, J.C. Herzog, and J.D. Guttman. Strand Spaces: Why is a Security Protocol Correct? In IEEE Conference on Security and Privacy, pages 160-171, 1998.

12. M. Fiore and M. Abadi. Computing symbolic models for verifying cryptographic protocols. In 14th IEEE Computer Security Foundations Workshop (CSFW'01), pages 160-173, Washington - Brussels - Tokyo, June 2001. IEEE.

13. J.-P. Jouannaud and C. Kirchner. Solving equations in abstract algebras: A rulebased survey of unification. In Jean-Louis Lassez and Gordon Plotkin, editors, Computational Logic: Essays in Honor of Alan Robinson. MIT-Press, 1991.

14. G. Lowe. A hierarchy of authentication specifications. In 10th IEEE Computer Security Foundations Workshop (CSFW' '77), pages 31-44, Washington - Brussels - Tokyo, June 1997. IEEE.

15. C. Meadows. Formal methods for cryptographic protocol analysis: Emerging issues and trends. IEEE Journal on Selected Areas in Communication, 21(1):44-54, January 2003.

16. J. Millen and V. Shmatikov. Constraint solving for bounded-process cryptographic protocol analysis. In ACM Conference on Computer and Communications Security, pages $166-175,2001$.

17. A. W. Roscoe. Intensional specification of security protocols. In 9th IEEE Computer Security Foundations Workshop (CSFW'96), pages 28-38, Washington Brussels - Tokyo, June 1996. IEEE.

18. M. Rusinowitch and M. Turuani. Protocol insecurity with finite number of sessions is NP-complete. In IEEE Computer Security Foundations Workshop, 2001.

19. S. Schneider. Verifying authentication protocols with CSP. In 10th IEEE Computer Security Foundations Workshop (CSFW'97), pages 3-17, Washington - Brussels Tokyo, June 1997. IEEE.

20. J. Thayer, J. Herzog, and J. Guttman. Honest Ideals on Strand Spaces. In IEEE Computer Security Foundations Workshop, pages 66-78, 1998.

21. Thomas Y. C. Woo and Simon S. Lam. Authentication for distributed systems. Computer, 25(1):39-52, January 1992. 